\title{
Switching the mechanism of mating type switching: a domesticated transposase supplants a domesticated homing endonuclease
}

\author{
Laura N. Rusche ${ }^{1}$ and Jasper Rine ${ }^{2,3}$ \\ ${ }^{1}$ Institute for Genome Sciences and Policy, and Biochemistry Department, Duke University, Durham, North Carolina 27708, \\ $\mathrm{USA}_{;}{ }^{2}$ California Institute for Quantitative Biosciences and Department of Molecular and Cell Biology, University of California \\ at Berkeley, Berkeley, California 94720, USA
}

Programmed DNA rearrangements are critical for the development of many organisms and, intriguingly, can be catalyzed by domesticated mobile genetic elements. In this issue of Genes \& Development, Barsoum and colleagues (pp. 33-44) demonstrate that, in the budding yeast Kluyveromyces lactis, a DNA rearrangement associated with mating type switching requires a domesticated transposase and occurs through a mechanism distinct from that in the related yeast, Saccharomyces cerevisiae. Thus, mechanisms for mating type switching have evolved multiple times, indicating the relative ease with which mobile genetic elements can be captured.

Biology is the recent beneficiary of remarkable technical advances that are flooding the field with terabytes of new DNA sequence information. This cornucopia of data raises the questions of what we are going to do with all this information and how we can best extract new biological insights. A study in this issue of Genes \& Development by Åström and colleagues (Barsoum et al. 2010) provides a beautiful illustration of the power of comparative genomics to illuminate new principles and research opportunities. The facile genetic manipulations available in yeast allow for the rigorous testing of hypotheses inspired by comparative genomics, which was an essential feature of this study since, at every turn, the results were wholly unanticipated. To put these elegant studies in context, we begin with some background on the biology of mating type switching in Saccharomyces cerevisiae, first described more than 50 years ago.

[Keywords: Mating type switch; transposable element; gene conversion; DNA double-strand break; sexual reproduction]

${ }^{3}$ Corresponding author.

E-MAIL jrine@berkeley.edu; FAX (510) 555-2768.

Article is online at http://www.genesdev.org/cgi/doi/10.1101/gad.1886310.

\section{Mating type switching in S. cerevisiae}

Mating type switching in S. cerevisiae, which is one of the best understood programmed DNA recombination events, allows haploid yeast cells of one mating type to produce haploid cells of the other type, thereby allowing sister cells to mate and become diploid. Diploid cells, in turn, can sporulate to produce haploid cells and offer yeast the advantages coincident with a sexual life cycle. In addition, diploid cells have certain selective advantages, such as enhanced DNA repair. However, the chief benefit of mating type switching may be its capacity to allow strains to purge their diploid genomes of deleterious recessive mutations by going through a transient haploid phase, with subsequent homozygous diploids generated from the viable haploid segregants. Given the importance of mating type switching for reproductive survival, traits that promote switching should be positively selected over evolutionary time. Interestingly, previous work in S. cerevisiae (Keeling and Roger 1995; Koufopanou and Burt 2005) and this new work in a related yeast, Kluyveromyces lactis, reveal that at least two different mobile genetic elements have been captured and harnessed for mating type switching. Because these domesticated mobile elements promote the reproductive success of the cells in which they reside, these elements proliferate in the population.

The mating type of haploid $S$. cerevisiae cells is determined by the MAT locus, which contains either the a 1 gene $(M A T a)$ or the $\alpha 1$ and $\alpha 2$ genes $(M A T \alpha)$. These genes encode transcription factors that determine the expression of cell-type-specific genes necessary for pheromone production and detection. S. cerevisiae cells also have extra copies of these mating type genes at the silenced $H M R \boldsymbol{a}$ and $H M L \alpha$ loci. These extra copies are not expressed, but are used as donors during the mating type switching process, in which a MATa cell can convert to a $M A T \alpha$ cell, and vice versa.

Switching in S. cerevisiae occurs through intrachromosomal gene conversion and relies on two blocks of 
conserved sequence that flank the MAT, HMR, and $H M L$ loci (for review, see Haber 1998). Homologous recombination between these loci is triggered by a double-strand break at $M A T$, generated by a site-specific endonuclease called HO. Because the recognition sequence for the HO endonuclease is within one of the conserved sequences, $M A T, H M L$, and $H M R$ all contain DNA sequences that could be cleaved by HO. However, the silenced chromatin at $H M R$ and $H M L$ blocks access of the endonuclease to these loci, so cutting occurs only at MAT. Once a doublestrand break is generated, this lesion is repaired using the general machinery required for homologous recombination. HMR or HML serve as donors, and MAT is the recipient of what is fundamentally a gene conversion event.

The HO endonuclease, which triggers switching, is expressed in late G1 in mother cells; i.e., those that have already budded off a daughter (for review, see Nasmyth 1993; Cosma 2004). After a single haploid spore germinates and divides, the new mother cell switches its mating type prior to DNA replication. Consequently, the next cell division produces two cells of the opposite mating type as the original spore. These two cells can then mate with the two cells produced from the first daughter, generating homozygous diploids. Thus, an isolated haploid spore will give rise to a clonal population of diploid cells. Consistent with this expectation, isolates of S. cerevisiae from the wild are often homozygous diploids (Replansky et al. 2008). This process does not occur in most laboratory strains because they contain a mutant version of the $H O$ gene (ho).

Interestingly, the $\mathrm{HO}$ protein evolved from homing endonucleases (Keeling and Roger 1995; Koufopanou and Burt 2005), a family of mobile genetic elements that replicate through a mechanism not unlike mating type switching (for review, see Gimble 2000; Burt and Koufopanou 2004). Homing endonucleases specifically cleave long recognition sequences and are found inserted in the middle of their own recognition sequences, effectively disrupting that sequence. In a diploid cell that is heterozygous for a homing endonuclease, the chromosome lacking the element becomes cleaved at the recognition site, and the broken chromosome is repaired by homologous recombination using the homing endonuclease-containing chromosome as a template. In this way, the homing endonuclease is copied to the repaired chromosome. The $H O$ gene is most closely related to $V D E$ (also known as PISceI), a homing endonuclease found in the TFP1 genes of $S$. cerevisiae and other yeast species. It is thought that a duplicated copy of $V D E$ acquired the specificity to cut at MAT rather than its own recognition sequence and was "domesticated" to facilitate mating type switching.

\section{Mating type switching in K. lactis}

Although mating type switching is well understood in $S$. cerevisiae, comparative studies examining the mechanism of switching in other related yeasts could illuminate how the advent of the $\mathrm{HO}$ endonuclease has changed the mechanism of mating type switching. In addition, comparative studies could reveal important aspects of switching that were missed in S. cerevisiae due to genes with overlapping functions.

The yeast $K$. lactis, which diverged from $S$. cerevisiae more than 100 million years ago, has several features that virtually insist on a comparative study of mating type switching. Like $S$. cerevisiae, $K$. lactis has an active $M A T$ locus and silenced HMR $\boldsymbol{a}$ and $H M L \alpha$ loci, all three of which are flanked by conserved blocks of sequence that would function admirably for a recombination-based mechanism of mating type switching. However, no functional $\mathrm{HO}$ endonuclease has been found in the K. lactis genome, although there is a highly degenerated pseudogene (Fabre et al. 2005). It has been presumed that mating type switching occurs through infrequent spontaneous homologous recombination (Butler et al. 2004), as in strains of $S$. cerevisiae lacking the $H O$ gene. However, an early study suggested that, in a lownutrient medium, mating type switching in $\mathrm{K}$. lactis occurred at a fairly high frequency (Herman and Roman 1966), implying that these conditions induce switching. But what would trigger switching in the absence of $\mathrm{HO}$ ?

Another interesting feature of $K$. lactis is that both $M A T \alpha$ and $H M L \alpha$ contain an additional gene, $\alpha 3$, that is absent in most other yeast species. This gene is necessary for $\alpha$ cells to mate (Åström et al. 2000), but, curiously, $\alpha 3$ function can be supplied from either MAT or HML. Its exact role in mating is still a mystery, as is the mechanism allowing its expression from the presumed silenced $H M L \alpha$ locus. This study highlights an entirely new function for $\alpha 3$, as discussed below.

A critical difference between $S$. cerevisiae and $K$. lactis is that mating (and perhaps mating type switching) occurs under different conditions in the two species. S. cerevisiae propagates in the wild primarily as diploid cells, and haploid cells mate efficiently almost as soon as they germinate in nutrient-rich conditions. $\mathrm{HO}$ endonuclease is expressed each time a cell enters G1 and commits to another cell cycle, ensuring that haploid cells will quickly become homozygous diploid cells. In contrast, K. lactis propagates primarily as haploid cells, with mating evident only when nutrients become scarce (Herman and Roman 1966; Schaffrath and Breunig 2000). Consequently, for K. lactis it would make sense that mating type switching, like mating itself, be induced by lownutrient conditions, as reported by Herman and Roman (1966). Thus, the regulatory network for switching in $K$. lactis is expected to be different from that of S. cerevisiae, but the extent of the differences, as revealed by the work of Åström and colleagues (Barsoum et al. 2010), was wholly unanticipated.

\section{Identification of a protein that stimulates mating type switching in both MATa and MATa K. lactis cells}

A fortuitous discovery provided Barsoum et al. (2010) with their first clue to understanding how mating type switching occurs in K. lactis. While conducting a screen for genes involved in silencing, Åström and colleagues (Barsoum et al. 2010) observed that overexpression of a 
$K$. lactis gene orthologous to RME1 (regulator of meiosis) from $S$. cerevisiae induced switching at high frequency. They subsequently named this gene MTS1 (mating type switch 1). This discovery revealed a regulated switching pathway in K. lactis, consistent with the observations of Herman and Roman (1966).

It is intriguing that both KIMTS1 and its ortholog, ScRME1, guide the response of haploid cells to lownutrient conditions in biologically appropriate ways. In haploid S. cerevisiae cells, Rme1 favors a foraging form of vegetative growth over meiosis, which would be catastrophic without two sets of chromosomes. In particular, this DNA-binding protein acts by repressing IME1, a master regulator of meiosis, and activating CLN2, a cyclin that promotes the transition from G1 to $S$ phase in the mitotic cycle (Blumental-Perry et al. 2002 and references therein). Although RME1 is constitutively expressed in haploid cells, it is further up-regulated upon nutrient depletion, a condition that would otherwise induce meiosis and sporulation. This increase in ScRmel results in the activation of genes whose expression promotes pseudohyphal formation and invasive growth (van Dyk et al. 2003). Presumably, this type of growth allows the yeast to grow toward nutrient-rich locations. In contrast, in diploid cells, RME1 is repressed and the cells can enter meiosis upon nutrient depletion.

In K. lactis, Mts1-like its ortholog ScRme1-has a role in guiding the response of haploid $K$. lactis cells to nutrient depletion. K. lactis haploid cells normally respond to this condition by mating in preparation for meiosis and sporulation (Herman and Roman 1966). However, in the absence of a mating type switching mechanism, a clonal population of haploid cells would be stuck without a mating partner. Fortunately, KlMts1 overcomes this obstacle by promoting switching, creating cells of opposite mating types that can mate to attain the exalted status of diploidy, at least until sporulation occurs. Consistent with this model, Åström and colleagues (Barsoum et al. 2010) demonstrated that KlMts1 is induced in low-nutrient conditions, explaining the initial observations of Herman and Roman (1966). It is not yet known whether KlMts1 also blocks meiosis in haploid cells, as ScRmel does, but a mechanism to delay meiosis until mating has occurred is certainly required.

To investigate how KlMts1 stimulates switching, Åström and colleagues (Barsoum et al. 2010) built on the observation that KlMts1, like its S. cerevisiae ortholog, has a three-zinc-finger DNA-binding domain, and they demonstrated that KlMts1 binds to sequences in both $M A T a$ and $M A T \alpha$. Importantly, deletion of these binding sites disrupts switching, indicating that the association of KlMts1 with $M A T$ is a critical step in mating type switching. Thus, KlMts1 has a role in switching analogous to that of $\mathrm{HO}$, which triggers switching in $S$. cerevisiae. Both KlMts1 and ScHO are induced under particular conditions (low nutrient or cell cycle phase) that are biologically appropriate for initiating switching followed by mating. In addition, both KlMts1 and ScHO recognize specific sequences at the target MAT loci. However, the analogy is limited, for, unlike HO, KlMts1 does not have an obvious nuclease domain, raising the question of how recombination is achieved.

\section{Switching from MATa to MATa in K. lactis requires the $\alpha 3$ gene}

Identifying the enzyme responsible for breaking the phosphodiester backbone at MAT to initiate recombination and mating type switching is the second major breakthrough made by Åström and colleagues (Barsoum et al. 2010), and is a big surprise. They found that the $\alpha 3$ gene, unique to $K$. lactis and its close relatives, is related to the mutator-like (MULE) family of transposases, suggesting that $\alpha 3$ may be involved in mating type switching. Indeed, switching is blocked in the absence of $\alpha 3$ or in

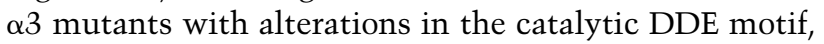
which is critical for function in this family of transposases. However, unlike Mts1, $\alpha 3$ is required only for switching from MAT $\alpha$ to MATa. Therefore, yet another protein must be responsible for driving the switch from MATa to MAT $\alpha$.

Curiously, the requirement for $\alpha 3$ in switching from $M A T \alpha$ to MATa cannot be satisfied by the $\alpha 3$ gene at $M A T \alpha$ ! Instead, $H M L \alpha$ is the essential source of $\alpha 3$ for this switch. This observation is very surprising because the copy of $\alpha 3$ at MAT is expressed, whereas the copy at $H M L$ is expected to be silenced. It thus appears that either $M A T \alpha 3$ is not expressed in low-nutrient conditions, or it is inactivated by the switching process itself (see below) prior to the time it must act. Moreover, $\alpha 3$ must be expressed from the silent $H M L$ locus, perhaps in a regulated manner. In fact, previous work is consistent with the notion that $H M L \alpha 3$ is expressed in low-nutrient conditions (Åström et al. 2000). Therefore, it is possible that $H M L \alpha 3$ may be induced under the same conditions that induce Mts1.

The mechanism by which $\alpha 3$ carries out mating type switching is beginning to emerge. The new work shows that induction of Mts1 results in the formation of a circular DNA containing the $\alpha 3$ gene. It is assumed that this circular DNA is derived from MAT $\alpha$ because deletion of the junction sequences in MAT $\alpha$ eliminated circle formation. In addition, these junction deletions eliminated switching, indicating that circle formation is a necessary intermediate in mating type switching. Åström and colleagues (Barsoum et al. 2010) propose, reasonably, that the formation of the circle causes a lesion at MAT that triggers the switching process. However, many questions about the mechanism of switching remain, including whether $\alpha 3$ is ever lost from $H M L$, whether the circle can insert elsewhere, what role Mts1 plays in initiating circle formation, and at what point in the process synapse formation with the donor $H M R \boldsymbol{a}$ locus occurs.

\section{Implications for regulation of silencing}

The observation that $\alpha 3$ is expressed from the silenced $H M L$ locus during mating type switching raises interesting questions regarding the regulation of the silencing 
mechanism. In rich medium, $H M L \alpha 3$ is repressed (Åström et al. 2000; Hickman and Rusche 2009), although it is not clear that it is completely off. This potential low level of expression may be sufficient for function, but it is also plausible that the minimal medium that induces mating type switching also induces $H M L \alpha 3$. If so, a mechanism must exist to overcome the silenced chromatin that forms at $H M L \alpha$. Such a mechanism has not been detected in S. cerevisiae, for which silencing of $H M L$ and $H M R$ by the Sir proteins appears to be constitutive, although Sir-mediated silencing at the telomeres is environmentally regulated in another yeast, Candida glabrata (Domergue et al. 2005).

It is also possible that relief of silencing in low-nutrient conditions is restricted to the $H M L$ locus. In a MATa cell, loss of silencing at HMRa would lead to the simultaneous expression of a and $\alpha$ genes, and hence a diploid identity, which would reduce mating. In this regard, it may be relevant that, in K. lactis, the mechanisms of silencing are distinct at HML and HMR (Hickman and Rusche 2009), and hence the two loci may be regulated independently.

A final question raised by the apparent expression of $H M L \alpha 3$ is how $H M L$ is protected from being the recipient of a mating type conversion. In S. cerevisiae, the assembly of Sir-mediated silenced chromatin at $H M L$ and $H M R$ protects these loci from cutting by the $\mathrm{HO}$ endonuclease. It remains unclear how $H M L \alpha 3$ can be expressed without also becoming a substrate for circle formation and switching.

\section{Switching from MATa to MATa in K. lactis occurs through a hairpin intermediate}

As described above, the mechanism of switching from MATa to MATa appears to be mechanistically distinct

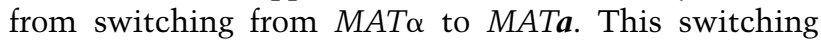
event is still triggered by the induction of Mts1, but does not require $\alpha 3$. So far, the enzyme responsible for initiating recombination has not been identified. However, one property of this mystery enzyme is the formation of hairpin-capped breaks at MATa. These breaks are reminiscent of intermediates in Rag1-mediated V(D)J recombination in immune cells. Like $\alpha 3$, Rag1 is thought to be derived from a transposase, and it is therefore plausible that yet another domesticated transposase is lurking in

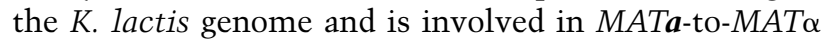
switching.

\section{Mobile genetic elements and programmed DNA rearrangements}

As noted by Åström and colleagues (Barsoum et al. 2010), their work adds to a growing list of cases in which mobile genetic elements have been adapted to facilitate programmed DNA recombination events (for review, see Volff 2006). For example, Rag1 is related to transposases in the transib superfamily (Kapitonov and Jurka 2005); PiggyMac, the enzyme responsible for elimination of germline sequences in the macronucleus of Paramecium, is derived from the PiggyBac family of transposases
(Baudry et al. 2009); and HO itself is derived from the $V D E$ family of homing endonucleases (Keeling and Roger 1995; Koufopanou and Burt 2005). Thus, the capture and harnessing of mobile genetic elements is becoming a common theme in programmed DNA rearrangements. Curiously, in the case of mating type switching, it appears that the capture of mobile genetic elements has occurred multiple times, with one element supplanting the previous one. K. lactis has a degenerate $\mathrm{HO}$ gene (Fabre et al. 2005), suggesting that HO played a role in mating type switching of an ancestor of $K$. lactis. However, the acquisition of the $\alpha 3$ gene from a MULE family transposase may have enabled switching to occur without $\mathrm{HO}$, resulting in its degeneration.

\section{Evolution of mating type switching in yeast}

The work of Åström and colleagues (Barsoum et al. 2010) calls for a revision of the prevailing model of the evolution of mating type switching. It has been proposed (Keeling and Roger 1995; Butler et al. 2004) that mating type switching, as it exists in $S$. cerevisiae, arose in two distinct steps. First, extra copies of the mating type loci, which could be maintained in a silenced state, were acquired. Such cassettes would allow switching at low frequencies through fortuitous homologous recombination. The second step involved the capture of a homing endonuclease that could trigger switching at high frequency by cutting MAT. This has been a compelling model, and is supported by observations that (1) multiple yeast species have extra, presumably silenced, mating type cassettes but lack the $\mathrm{HO}$ endonuclease; and (2) the presence of the $\mathrm{HO}$ endonuclease is phylogenetically restricted. However, the work of Åström and colleagues (Barsoum et al. 2010) calls into question the notion that strains lacking $\mathrm{HO}$ do not have mechanisms for inducing mating type switching, and also suggests that inducible mating type switching has been reinvented over the course of evolution.

In $K$. lactis, three separate proteins are required to achieve the same function as $H O$ in $S$. cerevisiae. The induction of Mts1 under low-nutrient conditions is required to initiate switching. In addition, $\alpha 3$ is required for recombination at $M A T \alpha$, and an as-yet-unidentified protein is required for recombination at MATa. Although $\alpha 3$ is unique to K. lactis and its close relatives, Mts1/Rmel is well conserved and could act as a trigger for switching in other species as well. In addition, the unknown protein required for the switch from MATa to MATa could be conserved and facilitate switching in other yeast. Moreover, currently unimagined switching mechanisms not found in $S$. cerevisiae or K. lactis may exist in other species. In fact, mating type switching appears to have evolved independently in the fission yeast Schizosaccaromyces pombe and occurs through yet another mechanism.

\section{Perspective}

This new work on mating type switching in K. lactis is a beautiful example of the ability of comparative genetic 
and genomic studies to reignite interest in problems that have been considered largely solved. By highlighting the evolutionary theme and variation among species, such studies lay out fascinating unanticipated opportunities that Åström's colleagues (Barsoum et al. 2010) and others will be harvesting in the coming years. Yet it is illuminating to consider how little of the story presented in this new study would be possible strictly by comparative genomics without the ability to manipulate the genome of $K$. lactis. For this reason, we anticipate that the easily manipulated genomes of microbes, and especially certain clades of fungi, will offer the opportunities for the deepest insights from comparative genomics in the near future. Hopefully, advances in targeted genome editing in animal cells, such as those provided by the zinc finger nucleases and other methods (Urnov et al. 2005; Bozas et al. 2009), will continue to be developed to allow the insights fueled by comparative genomics to lead to other studies as beautiful as that of Åström and colleagues (Barsoum et al. 2010).

\section{Acknowledgments}

We thank Stefan Åström, Meleah Hickman, and Joseph Heitman for critical reading of this manuscript. Research in the Rusche and Rine laboratories is funded by grants from the NIH: GM073991 (to L.R.) and GM31105 (to J.R.).

\section{References}

Åström SU, Kegel A, Sjostrand JO, Rine J. 2000. Kluyveromyces lactis Sir2p regulates cation sensitivity and maintains a specialized chromatin structure at the cryptic $\alpha$-locus. Genetics 156: 81-91.

Barsoum E, Martinez P, Åström SU. 2010. $\alpha 3$, a transposable element that promotes host sexual reproduction. Genes \& Dev (this issue). doi: 10.1101/gad.557310.

Baudry C, Malinsky S, Restituito M, Kapusta A, Rosa S, Meyer E, Bétermier M. 2009. PiggyMac, a domesticated piggyBac transposase involved in programmed genome rearrangements in the ciliate Paramecium tetraurelia. Genes \& Dev 23: 24782483.

Blumental-Perry A, Li W, Simchen G, Mitchell AP. 2002. Repression and activation domains of RME1p structurally overlap, but differ in genetic requirements. Mol Biol Cell 13: 1709-1721.

Bozas A, Beumer KJ, Trautman JK, Carroll D. 2009. Genetic analysis of zinc-finger nuclease-induced gene targeting in Drosophila. Genetics 182: 641-651.

Burt A, Koufopanou V. 2004. Homing endonuclease genes: The rise and fall and rise again of a selfish element. Curr Opin Genet Dev 14: 609-615.

Butler G, Kenny C, Fagan A, Kurischko C, Gaillardin C, Wolfe KH. 2004. Evolution of the MAT locus and its Ho endonuclease in yeast species. Proc Natl Acad Sci 101: 1632-1637.

Cosma MP. 2004. Daughter-specific repression of Saccharomyces cerevisiae HO: Ash1 is the commander. EMBO Rep 5: 953-957.

Domergue R, Castano I, De Las Penas A, Zupancic M, Lockatell V, Hebel JR, Johnson D, Cormack BP. 2005. Nicotinic acid limitation regulates silencing of Candida adhesins during UTI. Science 308: 866-870.

Fabre E, Muller H, Therizols P, Lafontaine I, Dujon B, Fairhead C. 2005. Comparative genomics in hemiascomycete yeasts:
Evolution of sex, silencing, and subtelomeres. Mol Biol Evol 22: 856-873.

Gimble FS. 2000. Invasion of a multitude of genetic niches by mobile endonuclease genes. FEMS Microbiol Lett 185: 99-107.

Haber JE. 1998. Mating-type gene switching in Saccharomyces cerevisiae. Annu Rev Genet 32: 561-599.

Herman A, Roman H. 1966. Allele specific determinants of homothallism in Saccharomyces lactis. Genetics 53: 727740.

Hickman MA, Rusche LN. 2009. The Sir2-Sum1 complex represses transcription using both promoter-specific and long-range mechanisms to regulate cell identity and sexual cycle in the yeast Kluyveromyces lactis. PLoS Genet 5: e1000710. doi: 10.1371/journal.pgen.1000710.

Kapitonov VV, Jurka J. 2005. RAG1 core and V(D)J recombination signal sequences were derived from Transib transposons. PLoS Biol 3: e181. doi: 10.1371/journal.pbio.0030181.

Keeling PJ, Roger AJ. 1995. The selfish pursuit of sex. Nature 375: 283.

Koufopanou V, Burt A. 2005. Degeneration and domestication of a selfish gene in yeast: Molecular evolution versus sitedirected mutagenesis. Mol Biol Evol 22: 1535-1538.

Nasmyth K. 1993. Regulating the HO endonuclease in yeast. Curr Opin Genet Dev 3: 286-294.

Replansky T, Koufopanou V, Greig D, Bell G. 2008. Saccharomyces sensu stricto as a model system for evolution and ecology. Trends Ecol Evol 23: 494-501.

Schaffrath R, Breunig KD. 2000. Genetics and molecular physiology of the yeast Kluyveromyces lactis. Fungal Genet Biol 30: $173-190$.

Urnov FD, Miller JC, Lee YL, Beausejour CM, Rock JM, Augustus S, Jamieson AC, Porteus MH, Gregory PD, Holmes MC. 2005. Highly efficient endogenous human gene correction using designed zinc-finger nucleases. Nature 435: 646651.

van Dyk D, Hansson G, Pretorius IS, Bauer FF. 2003. Cellular differentiation in response to nutrient availability: The repressor of meiosis, Rmelp, positively regulates invasive growth in Saccharomyces cerevisiae. Genetics 165: 10451058.

Volff JN. 2006. Turning junk into gold: Domestication of transposable elements and the creation of new genes in eukaryotes. Bioessays 28: 913-922. 


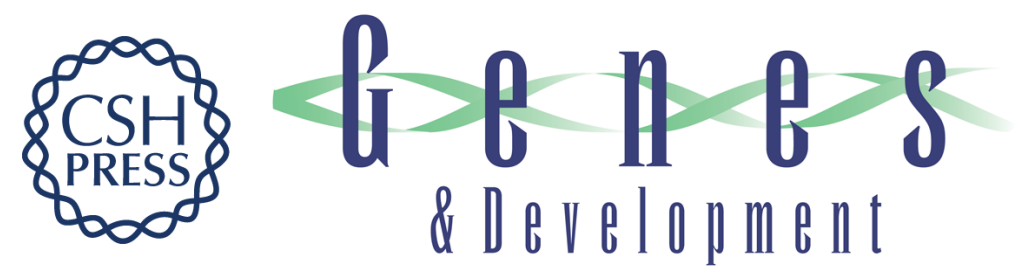

\section{Switching the mechanism of mating type switching: a domesticated transposase supplants a domesticated homing endonuclease}

Laura N. Rusche and Jasper Rine

Genes Dev. 2010, 24:

Access the most recent version at doi:10.1101/gad.1886310
Related Content
\pm 3 , a transposable element that promotes host sexual reproduction
Emad Barsoum, Paula Martinez and Stefan U. Åström
Genes Dev. January, 2010 24: 33-44
References This article cites 23 articles, 5 of which can be accessed free at: http://genesdev.cshlp.org/content/24/1/10.full.html\#ref-list-1
Articles cited in:
http://genesdev.cshlp.org/content/24/1/10.full.html\#related-urls
License
Email Alerting Receive free email alerts when new articles cite this article - sign up in the box at the top Service right corner of the article or click here.

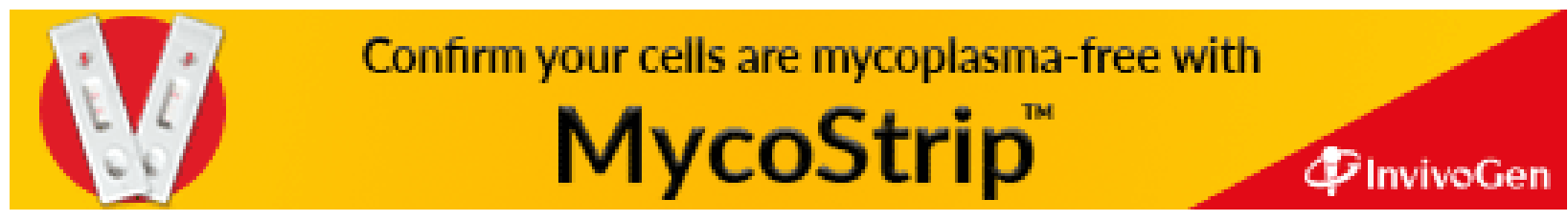

